\title{
Validating Data from Instruments in Harsh Environments: The Designer's Dilemma*
}

\author{
G. L. Johnson and E. E. Schultz \\ Nuclear Systems Safety Program \\ Lawrence Livermore National Laboratory
}

\begin{abstract}
Computer-based displays in nuclear power plants often validate data received from plant sensors and combine redundant data into a single reading. The Safety Parameter Display System is one example of such a system.

Unfortunately, many of the instruments that measure important plant parameters are exposed to extreme environments during a plant accident. These environments can induce large inşrument errors that current data validation and combination methods find difficult to handle.

We describe typical methods used to validate and combine data, illustrate the problems caused by large measurement errors, and suggest research areas where solutions to these problems might be found.
\end{abstract}

\section{INTRODUCTION}

Computer-based display systems are becoming more common in nuclear power plant control rooms. These systems improve plant safety by taking over rote operator tasks, which makes it possible for operators to focus more on operations. One such task is to examine redundant measurements of plant conditions and synthesize these into a single "best estimate" of the measured parameter's value. This process often includes data validation (i.e., resolving conflicts between multiple readings). The data validation algorithms currently used work well when measurement errors are expected to be small, but severe environmental conditions (associated with some plant accidents) can induce large measurement errors for important parameters. In certain circumstances, these large errors can cause data validation algorithms to present operators with misleading information. Future systems should use better methods that minimize this risk.

Before we discuss the problems that large errors cause in data validation, we will give a quick overview of data validation.

\section{Why Data Is Validated}

A reactor plant operator must make numerous decisions and act upon plant conditions as measured by installed instruments. For example, Pressurized Water Reactor (PWR) procedures require the operator to start feed-and-bleed cooling of the primary system if the steam generators are almost dry. To the operator, this condition is represented by steam generator water-level instruments indicating less than $5 \%$ of span.

As a rule, several instruments measure important conditions, so that a single failure does not result in the loss of important information. However, these redundant readings are often displayed in different parts of the control room to minimize common mode failure potential, so an operator must read several gauges and mentally resolve any conflicts between readings to determine the value of a critical plant parameter. In our example, a PWR often has as many as five indications of each steam generator's level. In addition, some of the instruments may be calibrated over different spans, and some may be calibrated for different operating conditions. Consequently, mental gymnastics are required to recognize and resolve conflicting readings. Although operators may not use all available displays, they would never base a judgment on a single reading. If one or more instruments fail on-scale (i.e., present an on-scale reading that differs from the actual value by more than the expected error), the operator faces a difficult task in deciding what value of level to use as the basis for decisions.

Computer-based displays can help in such cases. The computer can monitor the multiple readings, use some algorithm to resolve conflicting readings, and display a "best estimate" single value for a parameter. Providing the operator with a single value can significantly decrease work load and eliminate mistakes the operator may make in mentally resolving conflicting readings. Today, Safety Parameter Display Systems are one example of such a computer-based display. In the future, most control room information may be provided by computer systems that condense multiple measurements into a single value

*This work was supported by the United States Nuclear Regulatory Commission under a memorandum of understanding with the United States Department of Energy. 
for the operator's use. However, implementing this feature with current data validation techniques could cause more problems than it solves. To understand how this can happen, we must understand current data validation techniques. Note that in the following examples all errors are expressed in percent of instrument span.

\section{Data Validation Methods}

Data validation in most of today's Safety Parameter Display Systems is a two-step process. First, any readings that are outside the instrument's calibrated range are rejected. Second, consistency is checked between redundant readings, and those that are not consistent with the majority are rejected. The remaining valid readings are then averaged to generate a single value of the parameter for the operators' use. We'll confine our discussion to the second step-interchannel comparison. We'll use Safety Parameter Display System (SPDS) data validation methods for our examples.

Most SPDSs use one of three consistency-check methods:

(1) auctioneering,

(2) direct comparison, or

(3) comparison with the average.

Auctioneering simply selects the highest or lowest value, whichever is considered to be the most conservative. Figure 1 illustrates auctioncering for a three-input case. This method is readily fooled by single failures because it makes assumptions about which readings will lead operators to conservative actions. For these reasons, we believe auctioneering has limited usefulness in data validation for operator displays. NUREG1342 contains more discussion of the pitfalls and uses of auctioneering [1].

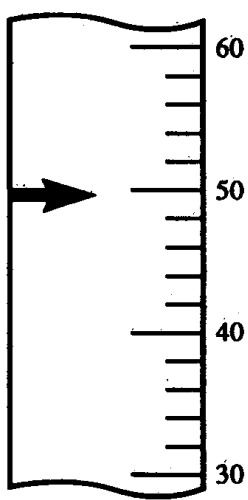

Input

Channel A

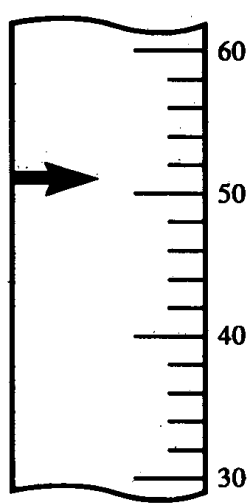

Input

Channel B
To check consistency, direct comparison rejects values if one or more differ from any of the others by more than some predetermined value. The most deviant value(s) are rejected and the remaining values are then averaged to produce the displayed value. To avoid unduly restricting the acceptance band, the acceptance criterion must be greater than the expected instrument error. If one assumes uniformly distributed random errors, and combines errors by the square root of the sum of squares, the expected deviation between two channels is less than the square root of the sum of the errors expected in any one channel. Thus if the maximum expected errors in the two channels are the same, the acceptance criterion may be as low as 1.4 times the expected error. Most systems, however, use twice the error. Common nuclear power plant instrument loops have errors between $\pm 2 \%$ and $\pm 4 \%$ of the instrument span. Therefore, direct comparison acceptance criteria are usually around $\pm 5 \%$. Figure 2 illustrates this method.

The third consistency-check method is comparison with the average. This method first averages all inputs. If any values differ from the average by more than the expected error, the most deviant input is rejected. The average and comparison is repeated until the algorithm generates an average that is within the acceptance criterion value of all inputs. This final average is then displayed. The acceptance criterion for this method can be equivalent to the expected instrument error. Figure 3 illustrates comparison with the average.

\section{The EFFects of Large ERrors}

The three illustrations show that two of the three comparison methods work reasonably well when measurement errors are small. Unfortunately, there are special cases in which properly functioning instruments have large errors.

Figure 1. Auctioneering selects the highest or lowest reading, whichever the designer predicts to be most conservative. Auctioneering does not attempt to eliminate failed on-scale readings; instead, it displays the "worst case" from various alternatives. In the example shown, the designer has determined that displaying the lowest value will always lead operators to take conservative action. 


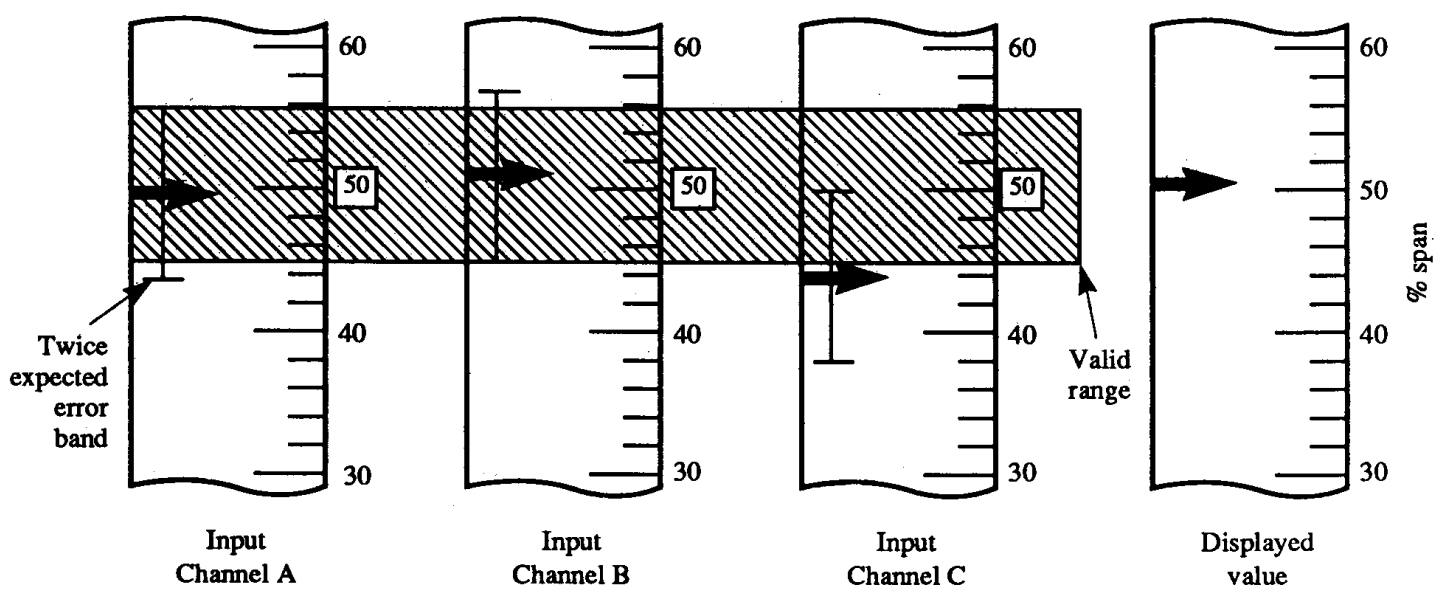

Figure 2. Direct comparison rejects the most deviant value(s) if the inputs differ from each other by more than some amount. The remaining values are usually averaged. In the example shown, the maximum expected errors are $\pm 3 \%$, so the validity criterion is that no channel may disagree with the others by more than $\pm 6 \%$. This combination of readings failed the validity test, so Input $C$ is omitted from the average because it differs from Inputs A and B by more than A and B differ from each other.

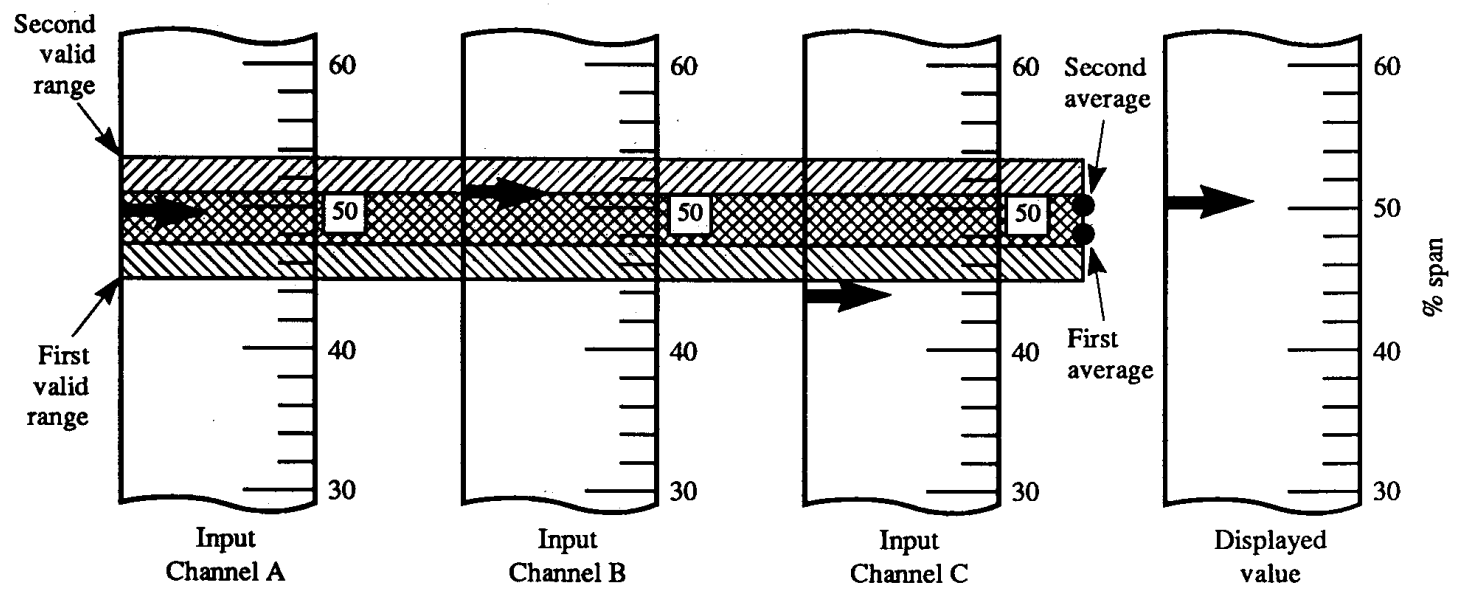

Figure 3. Comparison with the average rejects values that differ from the average by more than the validity criterion. If not all inputs pass on the first try, the input that disagrees the most is rejected, and the average-comparison process is repeated. In this example, direct comparison and comparison with the average produced the same displayed value.

An example of this is coolant system leaks in confined areas, which can subject instrument sensors and transmitters to a brutal environment. Instruments exposed to a loss-of-coolant accident in containment will be rapidly heated from their normal temperature of about $100^{\circ} \mathrm{F}$ to almost $400^{\circ} \mathrm{F}$. Steam blowdown will pressurize containment to between 50 and 70 psi. The differential pressure between the containment atmosphere and the inside of the instrument will try to force acidic steam into the instruments. At the same time, the instruments will be exposed to high radiation doses that can make insulation brittle, damage the structure of semiconductors, and further heat some components. Some instruments may eventually become submerged in hot, pressurized, acidic water.

Although some instruments are designed to survive such an extreme environment, testing shows that we can expect measurement errors of $\pm 10 \%$ to $\pm 20 \%$ under harsh environmental conditions. Let us examine how such errors affect data validation methods if we select acceptance criteria to avoid rejecting readings that disagree by less than the expected errors. We will, for the sake of illustration, discuss a hypothetical instrument channel for which the maximum expected error is $\pm 15 \%$. 
For the direct comparison method, we established that the acceptance criterion should be at least 1.4 times the expected error. This criterion is probably too tight for harsh environment cases because we're not sure that harsh-environment-induced errors are uniformly distributed. Consequently, to avoid rejecting valid readings, direct comparison validation should probably accept any readings that agree within twice the expected error. For our $\pm 15 \%$ instrument case, we would use $\pm 30 \%$ as the acceptable band; therefore, $60 \%$ of the instrument range would be within the valid band. Clearly, direct comparison is not an effective technique when expected errors are large.

The comparison-with-average method allows the use of tighter criteria: $\pm 15 \%$ in our example case. The acceptable band is still quite large. It is possible to envision cases in which the displayed average value can err by more than the expected instrument error. Consider, for example, the following case (illustrated in Figure 4). The true value of the parameter is $30 \%$ of the measuring instrument span, and three on-scale instruments are available to make the measurement. One instrument has failed and reads $65 \%$. The other two are working properly, but with errors on the high end of normal: one is $15 \%$ high; the other, $10 \%$ high.

In this example, the comparison-with-the-average algorithm would find all three readings valid, and operators would be presented a value that is in error by $20 \%$. We see that, in extreme cases, even the comparison-with-the-average method can confront operators with grossly erroneous data.

\section{THE DesigneR's DILEMma}

Large measurement errors present designers with a difficult choice in selecting data validation criteria.
A. The designer could set tight limits and risk rejecting valid data. This in itself can induce errors. Furthermore, it might lead the computer to determine that no valid measurements are available even when all the instruments are performing within expectation. As a result, operators would assume the burden of determining parameter values just when they least need additional tasks-during a severe accident.

B. The designer could select criteria that will not reject valid data, but then he/she risks biasing displayed values by even more than the expected measurement error. Biased readings can lead operators to take inappropriate actions. Consider, for example, the operator's response to a main steamor feed-line break in containment. A bias low in the steam generator water-level reading would cause the operator to prematurely initiate feed-and-bleed cooling of the primary system. Early initiation of feed-and-bleed cooling is undesirable because (1) it is more difficult to control than normal cooldown, and (2) it will contaminate-and possibly damage-equipment in containment.

Except in a few special cases, we advocate choice "B" in the belief that, while severe environments will cause large errors, on-scale failures that introduce large errors into averages will be relatively rare. But we find neither option attractive. Future control room computer systems need better data validation methods than those we have discussed.

\section{Possible Solutions}

There may be other options, however. We suggest that designers of future computer-based displays acknowledge the problems inherent in high-error measurements and consider some of the following approaches.

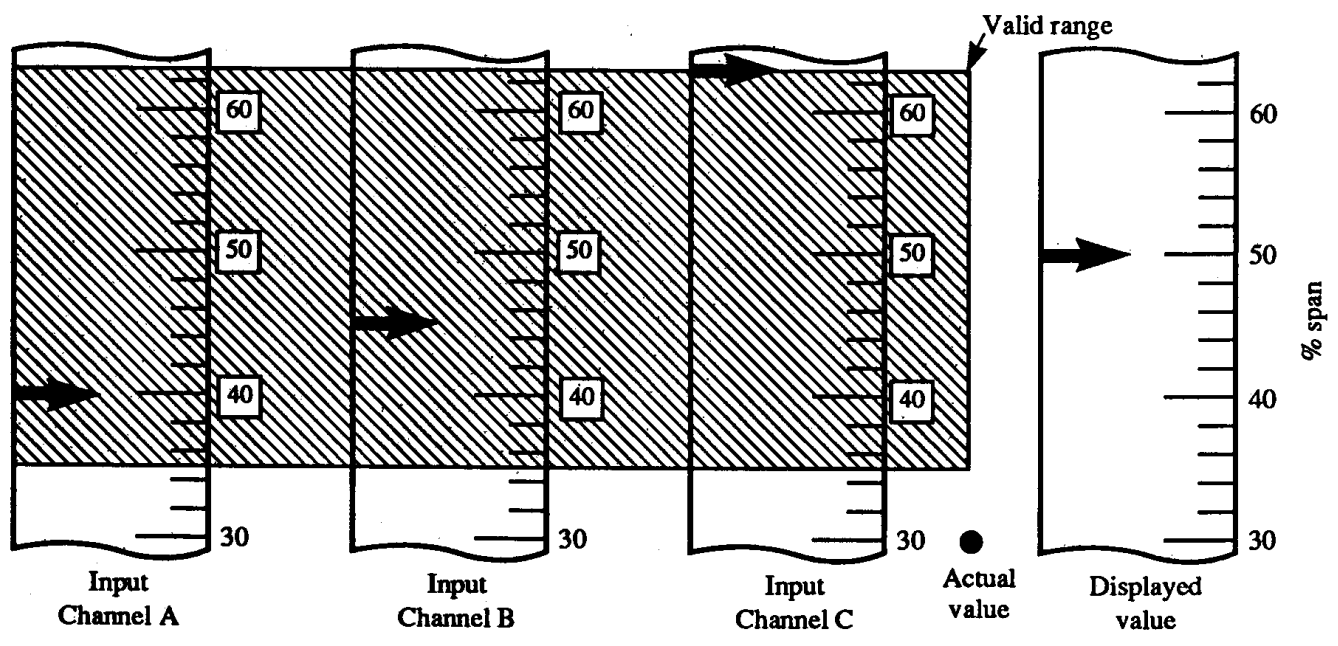

Figure 4. The consequences of large errors are serious. When expected measurement errors are large, validity criteria must be broad to avoid rejecting valid readings. This can, however, produce erroneous readings in extreme cases. In this example, large errors in two channels, together with a channel failed on-scale at the worst value, introduce a $20 \%$ error in the displayed value. We believe that future systems can-and should—do better. 
Improved Algorithms. Algorithms could be devised to ignore readings or to weight readings that are within the expected error band but disagree with the majority of readings. For example, the designer might take a weighted average of the inputs. One possibility might be to weight each valid input by the quantity $1-d / m$, where $d$ is deviation of the input from the unweighted average, and $m$ is maximum expected error. For our example in Figure 4, this would return a value of $46 \%$-about $4 \%$ better than the straight average.

Analytical Redundancy. Disagreements between instrument readings can be resolved by using knowledge about the process together with a different parameter to calculate a value of the parameter-of-interest for comparison. A simple example of analytical redundancy would be to compare a flow meter reading with the flow predicted by pump performance curves and pump speed. Several papers describe analytical-redundancy validation methods for data $[2,3,4]$. Although these methods are well known, the industry has not embraced them. We suspect that many designers think analytical-redundancy validation methods are expensive and unreliable. Investigation by the Electric Power Research Institute indicates that this is not the case [5].

Algorithms That Better Reflect Instrument Performance. We may not need to assume that harsh-environment errors are random and independent. Considerable documentation exists on instrument errors under harsh environmental conditions. We know of no formal attempt to analyze this body of information to determine if environmentally induced errors distribute themselves in ways that allow tighter validation criteria. If, for example, we could show that errors are usually in the same direction, or of the same magnitude, we could establish much tighter acceptance criteria.

Instrument-Health Monitoring. Comparison of readings is not the only available way to detect failed instruments. Thie, for example, suggests methods of detecting failed instruments by noise analysis [6]. Another possible approach is to design instruments that are self-monitoring. Assuming sensors are not the primary source of error, the designer might, for example, switch instrument transmitter inputs between the sensor and a known standard input to monitor the health of the transmitter.
Expert System Comparisons. We expect that a human operator would have done just as well with our example as our weighted average algorithm. Perhaps expert system methods would be more effective in validating data than algorithmic systems.

Instrument Relocation. New plants might be able to locate instrumentation such that the events to which instruments must respond do not expose the instruments to severe environments. This is probably the best option for instrumentation, but it may not be economically viable. It is certainly not a cost-effective option for existing plants.

\section{SUMMARY}

Data validation algorithms used in current nuclear power plant computer-based displays do not work well when large measurement errors are expected. We suggest that future computer-based displays need to deal more completely with this problem.

\section{REFERENCES}

[1] G. W. Lapinsky, Jr., R. J. Eckenrode, P. C. Goodman, R. P. Correia, A Status Report Regarding Industry Implementation of Safety Parameter Display Systems, NUREG-1342, U.S. Nuclear Regulatory Commission, Washington D.C., April 1989.

[2] C. P. Tzanos, "A Methodology for Validation of Measurements, Plant State Verification, and Fault Identification,"Nuclear Safety, Vol. 27, No. 3, pp. 351-358, July-September 1986.

[3] B. R. Upadhyaya, "Sensor Failure Detection and Estimation," Nuclear Safety, Vol.26, No. 1, pp. 32-43, January-February 1985.

[4] B. J. Benedict, S.S. Godbole, R. I. Lutz, O. L. Deutsch, Validation and Integration of Critical PWR Signals for Safety Parameter Display Systems, EPRI-NP-4566, Electric Power Research Institute, Palo Alto, Calif., May 1986.

[5] B. J. Benedict, R. I. Lutz, L. P. Smith, Validation of Critical Signals for the Safety Parameter Display System, EPRI-NP5066M, Electric Power Research Institute, Palo Alto, Calif., April 1987.

[6] J. A. Thie, "Surveillance of Instruments by Noise Analysis," Nuclear Safety, Vol. 22, No. 6, pp. 738-750, November-December 1981. 